\title{
YEPS: Running real-time experiments on a timesharing system
}

\author{
RICHARD G. PROUDFOOT \\ Yale University, New Haven, Connecticut 06520
}

\begin{abstract}
The Yale experimental programming system is described. This system consists of usercallable subroutines and a set of modifications to the UNIX operating system which support the development and execution of real-time psychology experiments. The interactions between a timesharing operating system and the provision of real-time facilities for experiments are discussed.
\end{abstract}

The Yale experimental programming system (YEPS) is designed to be an aid to an experimenter running a psychology experiment. The system consists of a library of user-callable subroutines and a set of modifications to the UNIX operating system. Together, these support the development and running of real-time psychology experiments.

The YEPS system runs on a PDP-11/40 computer, with 56k words of core memory and two 1.2-millionword disk drives. YEPS supports up to seven simultaneous users, each with his/her own CRT terminal. Thus, the system allows several subjects to participate in the same or different experiments concurrently, even while new experiments are being developed by other researchers. The amount of developmental work that can be done on the computer at any one time depends on the computational and real-time requirements of any experiments being run. The more stringent the real-time constraints of an experiment, the less timesharing of the processor can be allowed.

The system has recently been interfaced to digital logic hardware to allow the timing and control of perceptual experiments. In addition, YEPS has an A/D and D/A unit that allows it to control a Tektronix Model 604 display scope. The D/A unit also contains a crystal-controlled programmable clock that is used to time $\mathrm{I} / \mathrm{O}$ events.

UNIX is a general-purpose timesharing operating system developed at Bell Telephone Laboratories for the Digital Equipment Corporation PDP.11 computer family. The UNIX operating system makes available a large body of software, almost all of which is written in the $\mathrm{C}$ programming language.

$\mathrm{C}$ is a high-level programming language that provides convenient control structures, pointer variables, and user-defined data structures. The UNIX operating system kernel is written in $\mathrm{C}$ with the assistance of some assembler language routines. Learning to use and modify UNIX is greatly simplified because of the fact that UNIX is written in C. Our entire set of modifications to UNIX required that less than 50 lines of assembler code be written. The YEPS subroutine library and all psychology experiments are written in $\mathrm{C}$.

The $\mathrm{C}$ compiler can create a program composed of a pure procedure segment and a data segment. UNIX allows the sharing of pure procedure segments. This means that if several subjects are being run on the same experiment at the same time (as is frequently the case in our lab), experimenters can cut down on memory requirements by sharing a single pure procedure segment. However, there is one other possible kind of procedure sharing that UNIX does not support. This is the sharing of subroutine library functions. If these functions were shared there could be substantial additional memory savings, since most programs executed contain separate copies of the same subroutine functions.

UNIX supports a hierarchical file system on disk. When each user logs on, he/she automatically has access to his/her own directory of files. These files will contain the user's programs, data files, and test files. UNIX provides simple commands to change a user's directory, to copy files from one directory to another, to remove old files, and to create whole new directories.

A line-oriented text editor is provided, which is used for on-line development of programs. The editor can also be used to write instructions and examine data files. A text formatter is available to aid in writing papers and manuals. Also available is an interactive desk calculator program and a user mailbox facility to leave messages for others to read when they log on to the system. Altogether, UNIX provides more than 100 commands that can be executed from a user terminal. The user may even create a file containing a list of frequently repeated commands. This list may be "executed," causing the operating system to run each command in the file. Half a dozen new commands have been added during the development of YEPS. That so few new commands have been needed speaks well for the completeness of the original set.

The UNIX operating system makes available about 50 system calls that provide a large and flexible set 
of commands for the systems programmer. In building YEPS, we have changed UNIX by adding two new system calls and making changes to three others. The following section describes these modifications in some detail.

\section{YEPS}

The two new system calls added to UNIX are ACCUTIME and DELAY. ACCUTIME returns the time in milliseconds since January 1, 1970. DELAY(n) causes a pause for $n$ msec, after which the program issuing the delay is resumed. Since both ACCUTIME and DELAY use the system clock, which runs at $60 \mathrm{cps}$, the accuracy of these calls is, at best, in the range of $16 \mathrm{msec}$. UNIX provides the system calls TIME and SLEEP, which do roughly the same things as ACCUTIME and DELAY, but are only accurate to within $1 \mathrm{sec}$. In addition, SLEEP has the disadvantage that it allows a sleeping program to be swapped out to a disk. Since it may take a few seconds to swap the program back into core memory, the actual time at which the program is restarted may exceed the specified time by several seconds. One of the few disadvantages of using the entire software package provided with UNIX was the fact that SLEEP and TIME could not be changed without changing every program that calls them. This is why ACCUTIME and DELAY were added.

The three system calls that were modified are NICE, STTY, and READ. NICE is the system call that is used to set a user's job priority. It was modified to allow a user's job to lock itself into core. STTY is the system call that sets the terminal characteristics of the calling program. It was modified to allow an extra word of flag bits to be set. These extra bits are used to add more special-purpose terminal characteristics. For example, these flags were used in the implementation of the terminal timing features which will be described below. Finally, the READ system call was modified so that a READ call, with zero specified as the number of characters to be read, does not read any characters, but returns as its value the number of characters in the calling program's terminal input buffer. This feature is used to allow an experiment to present a stimulus and wait for a specified period of time. After the wait period is up, a zero-character READ call is issued. The presence of characters in the input buffer means the subject has responded and another READ call is then issued. This time the subject's response is actually read. If there are zero characters in the buffer, then the subject has not responded and some appropriate action, such as re-presenting the stimulus, can be taken.

\section{Input-Output Timing}

YEPS supports input-output (I/O) timing for terminals and terminal-type devices. This allows a precise time to be recorded when an output message is presented to a subject. A precise time can also be associated with every key a subject presses on the input keyboard. The modifications to the UNIX terminal drivers that allow it to support $1 / 0$ timing were originally designed and programmed by Robert Strom of Yale Computer Center.

The UNIX tty structure was extended with one word of flag bits: Two of these bits are used by the timing routines. One bit, the timing-enable bit, indicates that timing has been enabled for the terminal. When the timing-enable bit is first set, the timing clock is initialized and started. When the enable bit is on, writing the octal sequence 0176001 on that terminal causes the time to be echoed back into the input buffer and these two characters are never actually sent to the terminal. The format of the time that is echoed is as follows: an escape character (octal 0176), followed by octal 001 if an input time or octal 002 if an output time. Next come 4 bytes of binary time data, representing the time in tenths of milliseconds since the clock was started. This is followed by a terminating escape character. The second timing bit determines whether input characters will be associated with a time. When the timed bit is on, each input character is followed by a time in the format just described. These times record when the input keys were pressed. It is possible for a user to turn the timing bits on and off and process the time data directly; however, YEPS subroutine functions are provided so that a user need not be concerned with the details of the timing implementation.

Most of the YEPS library timing functions take as an argument a pointer, called the time pointer, which will hold 4 bytes of time data. Suppose timp is to be used as a time pointer. Then it should be declared as a four-element character array, as in Table 1, which demonstrates the ERASE ${ }^{1}$ function.

When the ERASE function is executed, it will place the 4 bytes giving the time the screen was erased into the array timp. Functions that take a time pointer as an argument will, if called with zero rather than a pointer [i.e., ERASE(0)], skip the step that records the time. The 4 bytes represent the number of tenths of milliseconds that have passed since clock initialization. This time by itself is not very meaningful, but

Table 1

The ERASE Function

char timp [4];

erase (timp); 
Table 2

Recording Input/Output Times

char timp1 [4], timp2[4], c, stimstring [80];

float $f$, latency ()

intimon( );

raw( );

display(stimstring, timp1, 1, 1, 5, 5);

$c=$ getctimed $($ timp 2$)$;

$\mathrm{f}=$ latency (timp1, timp2);

intimoff ();

noraw ();

when combined with other times that have been recorded, it may be used to compute a time interval. For example, the segment of a program in Table 2 displays a stimulus on a subject's terminal screen, recording a time. A time is also recorded when the subject presses any key in response to the stimulus. The two times may be used to compute a latency.

In Table 2, timp1 is used to hold the time the message is displayed and the time of the subject's response is recorded in timp2. The function LATENCY is used to compute a floating point number, which is the difference in seconds between the two times. The functions INTIMON and RAW are called to turn on input timing and to insure that a single response wakes up the calling program. Since the sequence of subroutine calls that display a message and record a response time are frequently used, they have been incorporated into the single function REACT, which is shown in Table 3. This program segment is identical in effect to the earlier one.

\section{String Handling and Data Manipulation}

In addition to the timing functions introduced above, the YEPS subroutine library contains a number of string and data manipulation functions. The YEPS set of string handling functions was designed to allow SNOBOL-like character string manipulation, but without the complexity of a full SNOBOL pattern matcher. To illustrate the use of these string functions, assume that during an experiment subjects are supposed to learn to associate names of people with a set of locations. For each name there is one associated location, and for each location there is one associated name. The experiment drills the subject on these associations in the following way: The program asks the subject, "Who was in the , where the blank location is to be supplied dynamically by the program. The subject responds by typing in the appropriate person's name. The program then examines the subject's response. If it is incorrect the program presents the right person's name and location. The segment from a YEPS program shown in Table 4 presents one practice trial for each of the locations.

The variable $\mathrm{n}$ shown in Table 4 is the number of persons and locations to be learned. The names are in the character array "names" and the locations are in the character array "locs." The following loop is executed for each of the $n$ locations. First, the terminal screen is erased (ERASE) and the message buffer is filled with blanks (BLANKCHR). Next, the message "Who was in the" and the appropriate location are moved into the message buffer (COPYWD), any extra blanks between the words in the buffer are removed (RMXBLANKS), and the question is then displayed on the subject's terminal (DISPLAY). The subject types in a name. If this name matches (SUBSTR) the correct name, the message "CORRECT" is displayed

Table 3

The REACT Function

float $\mathrm{f}$;

char $c$, stimstring [80];

$c=$ react(stimstring, \&f, $1,1,5,5)$;

Table 4

A Name and Location Learning Drill

int $\mathbf{i}$;

char names[n] [20], locs[n] [20];

char buf $[80]$;

for $(\mathrm{i}=0 ; \mathrm{i}<\mathrm{n} ; \mathrm{i}++)$

blankchr (buf, 79);

copywd ("WHO WAS IN THE", buf);

copywd (\&locs[i] [0] , \&buf [20]);

rmxblanks (bufs, 79);

display (buf, $0,1,1,1,1$ );

gets (buf); $\left.\quad\right|^{*}$ read subject's response */

if (substr(buf, \&/names[i] [0] $>=0$ )

\{

display ("CORRECT", 0, 1, 3, 1, 3); sleep (2);

else

\{

display ("WRONG", $0,1,2,1,2)$;

blankchr (buf, 79);

copywd (\&names[i] [0], \&buf [0]);

copywd ("WAS IN THE", \&buf [20]); copywd (\&locs[i] [0], \&buf [35]); rmxblanks (buf, 79); display (buf, $0,1,3,1,3$,); sleep (2); 
on the subject's terminal. If the names do not match, the message "WRONG" is displayed and the correct name and location are placed in the message buffer and displayed.

\section{REAL-TIME TASKS}

A task is defined as real-time if it requires that certain process events occur at times synchronized with an external clock. To be more specific, we will assume that the real-time system contains two operating system primitives called GETTIME and DELAY. GETTIME() returns the current time (in milliseconds). $\operatorname{DELAY}(\mathrm{n})$ causes the process to suspend activity for n time units from the current time, after which the program statement following the DELAY will be executed. We assume that both GETTIME and DELAY have negligible overhead. Also, both primitives are assumed to be measured in the same units and to be correct within certain tolerances. If more than one task is being processed simultaneously, it is assumed that interruptions of a task occur only when that task is in a delay interval. The fragment of code presented in Table 5 typifies a real-time task. (The following approach as well as others are discussed in Kaplan, 1977.) In this task, a message is to be presented for exactly $500 \mathrm{msec}$. During this time the program must perform some computation (perhaps to select the next message to present). After this computation, a delay for the amount of time remaining in the 500-msec interval is issued. The real-time constraint is enforced by the requirement that DELAY must be called with a nonnegative argument. If the argument to DELAY is negative, it would be reasonable to have the operating system report that an error had occurred.

Timesharing is defined to be the allocation of CPU time and other computer resources among a group of users so that each user seems to have a dedicated computer to work on. In a timesharing system, CPU time is usually allocated among the users in small pieces so that computer response time is short for requests requiring little computation. In practice the size of the work load on a timesharing system may be very apparent to the users in terms of hesitation and slowed systemresponse times.

\section{Real-Time vs. Timesharing}

There are some incompatibilities between providing timesharing service to some users while at the same time providing real-time service to other users. Because the amount of random-access memory on a computer system is constrained both by cost and by hardware limitations, a timesharing system frequently uses a disk for program swapping or paging. When a process is swapped out of main memory, its response time to an external event is on the order of seconds-far too long for most real-time requirements. Thus, a timesharing
Table 5

Prototype of a Real-Time Task

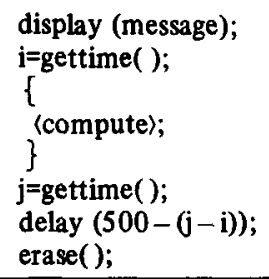

system that might swap out a process with real-time constraints must be modified so that this swapping does not take place. A second source of incompatibility is in the allocation of CPU time and other resources among timesharing processes. To meet real-time requirements, a program must sometimes retain control of the CPU until a computation is complete. This conflicts with the timesharing goal of quick response to programs with trivial computational requirements, since they may be locked out of the CPU for a considerable interval while real-time requirements are being satisfied.

A real-time task can be represented by two numbers: $D(M)$ represents the required elapsed time between external events of task $M$; $P(M)$ represents the time required to do the computation associated with task $M$. [As will be shown shortly, $\mathrm{P}(\mathrm{M})$ is sometimes an estimate of the time required to complete execution of $\mathrm{M}$ ] . In the prototype real-time task shown in Table 5, $P$ is the time required to execute the compute block and $D$ is the 500 -msec interval between presentation of the message and its erasure. A real-time program consists of a sequence of such real-time tasks, perhaps interspersed with computations for which there is no real-time constraint (in this case we might assume that $\mathrm{D}$ is infinite). There is no need for the compute block to be executed at the beginning of the interval $D$. We only require that the computation be completed before the interval $D$ elapses. At any point in the interval $D$, precise values for the time remaining in the $D$ and $P$ intervals can be determined. Thus, the real-time task can be segmented at arbitrary points in time.

Where a single processor is timeshared among several real-time tasks, it can be modeled as $\mathrm{n}$ ordered pairs, $(P[1], D[1]) \ldots(P[n], D[n])$, at a single instant, $t$, in time. The problem confronted by the timesharing operating system is that of scheduling the processor in such a way that all constraints at time $t$ plus delta $t$ will be met; or, failing this, that the scheduler will send out an error message and meet the maximum possible number of constraints. If the availability of the processor over time delta $t$ is viewed as a container of size delta $t$, and the constraining computations of the $n$ processes are viewed as objects of volume $P[i]$, then the scheduling problem is reduced to finding the optimum way of packing $n$ objects of size $P[i]$ in 
space delta t. This is the well known knapsack-packing problem. Such problems belong to the class called NP-complete problems (Karp, 1972), conjectured to require exponential time (as a function of $n$ ) to solve. This means, in effect, that it may take our processor an inordinate amount of time to compute the appropriate order in which to execute the constraining computations.

If rather than requiring that the constraints be satisfied to the optimum extent we require them only to be within epsilon of the optimum, then there exist $\mathrm{O}\left(\left(\mathrm{n}^{*} \log (\mathrm{n})\right) / \mathrm{epsilon}\right)$ algorithms to schedule the $\mathrm{n}$ real-time processes (Karp, 1976). The $P[i]$ times are likely to be estimates or mean values rather than the fixed and exact quantities we have been assuming. Since there will be some variation in the $P[i]$ s anyway, it seems reasonable to require only that the scheduler be within epsilon of the optimum schedule.

Meeting the real-time requirements of psychology experiments has not been a problem under our normal operating conditions. Intuitively, it seems clear that if all the real-time computations that must be done require much less time than the intervals between the external events whose duration is to be controlled, the scheduler should have little trouble meeting the real-time requirements. More formally, if the sum of the $P[i] s$ is much less than the minimum of the $D[i] s$, then the operating system should have no difficulty completing all the computations without violating the real-time constraints. In actual operation of the YEPS system, we have found that less than $10 \%$ of the available CPU time is being used. Much of the CPU time being used is consumed in the development of new experiments and the running of analysis programs, neither of which have any real-time requirements. Thus, a sophisticated scheduler is not needed in normal operation.

\section{Determining Computation Times}

The foregoing discussion assumes that the scheduler has available an accurate computation time for each real-time task (the $\mathrm{P}[\mathrm{i}] \mathrm{s}$ ). These times must be available before the scheduler can decide on the best order in which to do the tasks. We must now consider how these execution times might be derived. This will be done with a view toward determining the feasibility of building a very knowledgeable compiler that could examine a program and determine the execution times of the various segments of that program.

Each statement in a high-level language such as $\mathrm{C}$ can be considered as being of one of four types. The first type of statement is a straight-line-code statement. These statements include simple assignments and arithmetic computations but do not include any control flow statements or function calls. The second type of statement is an operating system call. Operating system calls can be analyzed into their component instructions. In practice, operating system calls are used for many purposes, including opening and closing files, changing job priorities, creating new processes, and so on. Typically, operating system calls are I/O statements allowing a program to communicate with the outside world. To simplify matters, we assume that there are four system calls: READ, WRITE, DELAY, and GETTIME. An operating system call often imposes real-time constraints on a program. For example, a write to a slow device (such as a Teletype) may actually require a few seconds for outputting a message.

The third type of statement is a control flow statement. These are statements such as IF-THEN-ELSE, WHILE, FOR, GOTO. In control statements there is always a Boolean expression that is either true or false when it is evaluated. The control statement specifies two separate statements or sets of statements to be executed according to whether or not the Boolean evaluates to true or false. Any program containing the control statements WHILE, FOR, and GOTO can be transformed into an equivalent program consisting of a possibly infinite binary tree structure, in which the nodes represent IF-THEN-ELSE statements, and whose arcs contain only straight-line-code and operating system calls. At any node, the left-hand branch is the code that is executed if the Boolean is false and the right-hand node will be executed if it is true.

The final type of statement is a function call. Function calls can be analyzed as if they were macros; that is, as if everywhere a function call occurs it is replaced by the function's defining code. If the value returned by a function is used in a computation or assignment, we assume that the last statement of the function code is the assignment of the function's value to an appropriately declared variable. This variable is then referenced whenever the function's value is required. If all function calls in a program are uniformly replaced by their defining code, the resulting program consists solely of the other three types of statements.

With each statement, $S$, in a program, there is associated a time function, $T(S)$, which gives the time it takes the computer to execute statement $S$. The function $T$ returns an exact value for each type of statement except system calls. Since system calls operate under real-time constraints (such as disk seek time and data transfer rates) that depend dynamically on the state of the operating system, it is impossible to give an exact time that it will take to execute a system call. We therefore assume that the function $\mathrm{T}$, when its argument is a system call, returns an average value and that statistical information about the distribution of times of operating system calls has been compiled from records of prior operating system calls.

In the case of control flow statements, the evaluation of each Boolean takes a fixed amount of time, which can be precomputed at compile time. However, whether the left or right branch of the IF-THEN-ELSE node is executed depends dynamically on the value of the Boolean expression.

A program execution is defined as a path through 
the program tree from the root node to one of its leaf nodes. A real-time task is again represented by the two numbers $P$ and $D$. The point where the timing begins for the interval $D, T[$ initial], can be located in the tree representation of the program containing the realtime task. The end of the interval D can occur at many (perhaps infinitely many) locations in the program tree, depending on the truth value of the Booleans in any intervening IF-THEN-ELSE nodes. A real-time task falls into one of three classes: (1) At $T$ [initial] all Booleans in the path from the start of the real-time interval to its end are defined and their values will not change before they are evaluated in their associated IF-THEN-ELSE statements. In this case an exact time (within the statistical range of any intervening system calls) can be computed for the execution time, $P$, associated with the real-time task. The discussion of the scheduling of real-time tasks assumed that all real-time tasks were of this first type. (2) One (or more) of the Booleans on the path(s) to end of the real-time task depends in a complex way on the computation being done in the task. In this class, only a worst-case upper bound of the execution time $P$ is available when the real-time task is started. For example, a Boolean may depend on the result of a SUBSTRING operation on the data in an input buffer. In this case, execution time $P$ can be bounded by the product of the length of the input buffer times the length of the substring being searched for, but the exact time is dependent on the data in such a way that to compute it requires that the SUBSTRING algorithm be run. (3) One or more of the Booleans on the path(s) to the end of the real-time task depends on input (a READ system call) that is done within the real-time interval itself. This case corresponds to a loop in the program, where the loop is only exited if a particular input character is read. Such a loop can potentially be infinite.

Given that an execution time is associated with each language statement and that real-time tasks can be divided into the three classes defined above, one can imagine building a compiler that knows about statementexecution times and operating system call times, and which could classify real-time tasks according to the above scheme. The compiler would compute an execution time for Class 1 real-time tasks, give worstcase bounds for Class 2 real-time tasks, and identify the input dependencies of Class 3 tasks. If for Class 3 tasks the programmer is allowed to specify the range and distribution of input data, then estimates of the computation time required for these tasks could be given. Such a compiler would be very helpful in determining if a real-time task is feasible and in allowing a programmer to change a program so that its real-time requirements are more likely to be met.

\section{CONCLUSION}

YEPS as a timesharing system has been successful in handling a broad range of experimental tasks with differing timing requirements. Provision is made for running those experiments on a stand-alone basis whose real-time requirements are very stringent. Most of our real-time requirements can be met without sacrificing the advantages of a timesharing system.

\section{REFERENCES}

KAPLAN, H. L. Clock-driven FORTRAN task scheduling in a multiprogramming environment. Behavior Research Methods \& Instrumentation, 1977, 9, 176-183.

KARP, R. M. Reducibility among combinatorial problems. In R. E. Miller \& J. W. Thatcher (Eds.), Complexity of computer computations. New York: Plenum Press, 1972. Pp. 85-104. KARP, R. M. The probabilistic analysis of combinatorial search alogrithms. In J. F. Traub (Ed.), Algorithms and complexity: New directions and recent results. New York: Academic Press, 1976. Pp. 1-19.

\section{NOTE}

1. A number of YEPS functions such as ERASE and DISPLAY are the same in name and/or action as commands in the General Experiment Programming System which was developed at the University of Michigan Human Performance Center. The implementation of these commands is entirely different. 